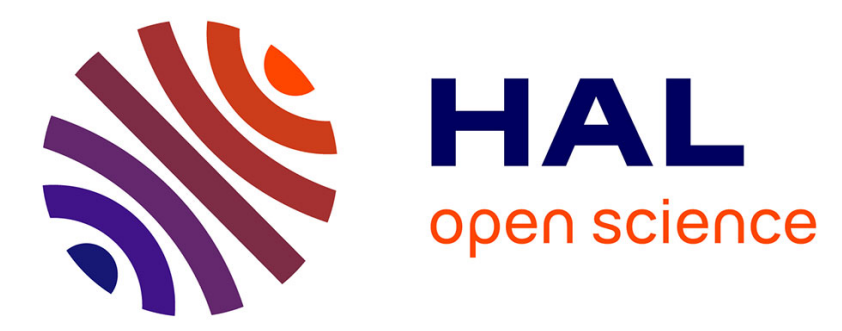

\title{
Experimental study of stratified turbulence forced with columnar dipoles
}

\author{
Pierre Augier, Paul Billant, M. E. Negretti, Jean-Marc Chomaz
}

\section{To cite this version:}

Pierre Augier, Paul Billant, M. E. Negretti, Jean-Marc Chomaz. Experimental study of stratified turbulence forced with columnar dipoles. Physics of Fluids, 2014, 26 (4), pp.046603. 10.1063/1.4870703 . hal-01048580

\section{HAL Id: hal-01048580 \\ https://hal-polytechnique.archives-ouvertes.fr/hal-01048580}

Submitted on 25 Jul 2014

HAL is a multi-disciplinary open access archive for the deposit and dissemination of scientific research documents, whether they are published or not. The documents may come from teaching and research institutions in France or abroad, or from public or private research centers.
L'archive ouverte pluridisciplinaire HAL, est destinée au dépôt et à la diffusion de documents scientifiques de niveau recherche, publiés ou non, émanant des établissements d'enseignement et de recherche français ou étrangers, des laboratoires publics ou privés. 


\section{AIP | Physics of Fluids}

\section{Experimental study of stratified turbulence forced with columnar dipoles}

P. Augier, P. Billant, M. E. Negretti, and J.-M. Chomaz

Citation: Physics of Fluids (1994-present) 26, 046603 (2014); doi: 10.1063/1.4870703

View online: http://dx.doi.org/10.1063/1.4870703

View Table of Contents: http://scitation.aip.org/content/aip/journal/pof2/26/4?ver=pdfcov

Published by the AIP Publishing

\section{Articles you may be interested in}

Turbulent flow in rib-roughened channel under the effect of Coriolis and rotational buoyancy forces

Phys. Fluids 26, 045111 (2014); 10.1063/1.4871019

On the rising motion of a drop in stratified fluids

Phys. Fluids 25, 103302 (2013); 10.1063/1.4823724

The spatial evolution of fluctuations in a self-propelled wake compared to a patch of turbulence

Phys. Fluids 25, 095106 (2013); 10.1063/1.4819877

Experimental study of temperature fluctuations in forced stably stratified turbulent flows

Phys. Fluids 25, 015111 (2013); 10.1063/1.4775380

Effect of the Prandtl number on a stratified turbulent wake

Phys. Fluids 22, 095102 (2010); 10.1063/1.3478841

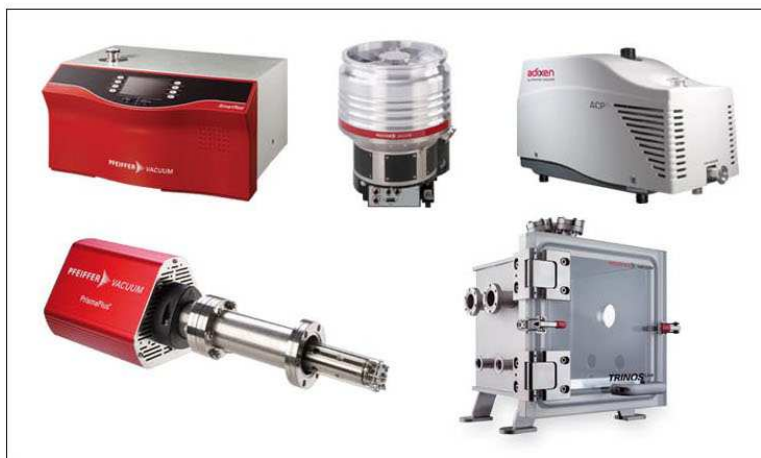

Vacuum Solutions from a Single Source

- Turbopumps

- Backing pumps

- Leak detectors

- Measurement and analysis equipment

- Chambers and components 


\title{
Experimental study of stratified turbulence forced with columnar dipoles
}

\author{
P. Augier, a) P. Billant, M. E. Negretti, ${ }^{\text {b) }}$ and J.-M. Chomaz \\ LadHyX, CNRS, Ecole Polytechnique, 91128 Palaiseau Cedex, France
}

(Received 22 November 2013; accepted 17 March 2014; published online 14 April 2014)

\begin{abstract}
We present a novel experimental setup aimed at producing a forced strongly stratified turbulent flow. The flow is forced by an arena of 12 vortex pair generators in a large tank. The continuous interactions of the randomly produced vortex pairs give rise to a statistically stationary disordered flow in contrast to previous experiments where the stratified turbulence is decaying. The buoyancy frequency $N$ is set to its highest value $N=1.7 \mathrm{rad} / \mathrm{s}$ using salt as stratifying agent so that the horizontal Froude number $F_{h}=\Omega / N$ is low, while the buoyancy Reynolds number $\mathcal{R}=R e F_{h}{ }^{2}$, where $R e=$ $\Omega a^{2} / v$ is the classical Reynolds number, is as high as possible given the experimental constraints $(\Omega$ is the maximum angular velocity of the vortices, $a$ their radius and $v$ the viscosity). PIV measurements show that the flow is not homogeneous in the horizontal plane and is organised into horizontal layers along the vertical. When $\mathcal{R}$ is increased, we observe a progressive evolution from the viscosity dominated regime with smooth layers to a regime with small scales superimposed on the layers and for which the vertical Froude number is of order one. The latter regime resembles the strongly stratified turbulent regime with a downscale cascade that has been predicted for large $\mathcal{R}$. However, horizontal second order structure functions do not exhibit a clear inertial range for the largest $\mathcal{R}$ achieved $\mathcal{R}=310$. In addition, the corresponding turbulent buoyancy Reynolds number $\mathcal{R}_{t}=P /\left(v N^{2}\right)$ based on an estimation of the injection rate of energy $P$ is only of order unity $\mathcal{R}_{t} \simeq 0.4$ indicating that only the edge of the strongly stratified turbulent regime has been reached. However, these results suggest that sufficiently large turbulent buoyancy Reynolds numbers, $\mathcal{R}_{t} \simeq 10$, could be achieved experimentally by scaling up five times this novel set-up. (c) 2014 AIP Publishing LLC. [http://dx.doi.org/10.1063/1.4870703]
\end{abstract}

\section{INTRODUCTION}

Atmospheric mesoscales $\left(5 \mathrm{~km} \lesssim L_{h} \lesssim 100 \mathrm{~km}\right)$ and oceanic submesoscales $\left(100 \mathrm{~m} \lesssim L_{h}\right.$ $\lesssim 5 \mathrm{~km}$ ) are weakly influenced by rotation and strongly influenced by stable density stratification. ${ }^{1}$ In other words, these scales are characterized by a relatively large Rossby number $R o=U /\left(f L_{h}\right)$ and a low horizontal Froude number $F_{h}=U /\left(N L_{h}\right)$, with $U$ and $L_{h}$ representing the characteristic horizontal velocity and length scales, $N$ is the Brunt-Väisälä frequency, and $f$ is the Coriolis parameter.

The structure of such strongly stratified flows is characterized by thin horizontal layers. ${ }^{2}$ This strong anisotropy implies that vertical gradients are high and play an important role in the dynamics. Vertical advective transport and vertical viscous diffusion act with two different characteristic length scales: respectively, the buoyancy length scale $L_{b}=U / N$ and the viscous length scale $L_{v}=\sqrt{v L_{h} / U}$, where $v$ is the viscosity. The ratio between these two length scales is related to the buoyancy

\footnotetext{
a) Present address: Department of Applied Mathematics and Theoretical Physics, University of Cambridge, CMS, Wilberforce Road, Cambridge, CB3 0WA, United Kingdom. Electronic mail: p.augier@ damtp.cam.ac.uk.

b) Present address: LEGI, CNRS/INPG, 38041 Grenoble Cedex 9, France.
} 
Reynolds number $\mathcal{R}=\left(L_{b} / L_{v}\right)^{2}=R e F_{h}{ }^{2}$, where $R e=U L_{h} / v$ is the classical Reynolds number. ${ }^{3-5}$ For geophysical flows, the buoyancy Reynolds number is typically very high even if $F_{h}$ is small, whereas it is difficult to establish a high buoyancy Reynolds number in laboratory experiments of strongly stratified flows. ${ }^{6}$

The layered structure can arise spontaneously through a generic instability, the zigzag instability, when vertical vortices interact. ${ }^{7-9}$ This instability slices columnar vortices into "pancake" vortices with a vertical length scale $L_{v}$ of the order of the buoyancy length scale so that their aspect ratio $L_{v} / L_{h}$ scales like $F_{h}$. Theoretically, the scaling law $F_{v}=U /\left(N L_{v}\right) \sim 1$ derives from an invariance of the hydrostatic Boussinesq Euler equations that is valid in the limits $F_{h} \ll 1$ and $\mathcal{R} \gg 1 .{ }^{3}$ This scaling law suggests the possible development of instabilities. The Richardson number $R i \simeq 1 / F_{v}{ }^{2}$ is indeed of order one, which means that layers and pancake structures are potentially unstable to the shear instability. Similarly, the density perturbations are of the same order as the mean density gradient when $F_{v} \sim 1$ meaning that gravitationally unstable regions may appear. ${ }^{10-12}$ Lindborg $^{13}$ proposed an universal regime of strongly stratified turbulence associated with a stratified downscale energy cascade and a horizontal kinetic energy spectrum equal to $E\left(k_{h}\right)=C_{1} \varepsilon_{K}{ }^{2 / 3} k_{h}^{-5 / 3}$, where $k_{h}$ is the horizontal wavenumber, and $C_{1} \simeq 0.5$ is an universal constant.

This theory of strongly stratified turbulence explains many numerical results ${ }^{4,5,13-16}$ and is compatible with many in situ observations. ${ }^{1}$ However, it has never been confirmed in laboratory experiments and it does not explain all the observations in numerical simulations, such as the "bump" in the horizontal spectra observed at relatively small scales. ${ }^{5,17,18}$ In addition, most of the simulations on forced stratified turbulence are performed using pseudo-spectral methods with periodic boundary conditions, characterized by the absence of walls. In these simulations, a large amount of energy accumulates in horizontally invariant, vertically sheared modes called "shear modes." ${ }^{19}$ These modes are not well understood and various explanations have been given for their existence. Smith and Waleffe ${ }^{19}$ have explained the growth of the shear-mode energy by off-resonant three waves interactions. In contrast, Lindborg ${ }^{13}$ argues that the continuous increase of the energy in the shear modes observed in many numerical simulations is due to a viscous effect. Nevertheless, even for the simulations with large $\mathcal{R}$, the portion of energy in those modes is quite high, for example, it represents $\sim 25 \%$ of the total energy in the study of Brethouwer $\mathrm{et} \mathrm{al.}{ }^{5}$ Thus, it is likely that these shear modes influence all the cascade via highly non-local interactions in most of the numerical simulations. ${ }^{20}$

In contrast, such shear modes have not been reported in experiments but the main challenge to produce stratified turbulence in the laboratory is the fact that dissipation at large horizontal scales dominates the dynamics when the buoyancy Reynolds number is small even if the Reynolds number is large. For example, Praud et al. ${ }^{6}$ have shown that experimental decaying grid turbulence in a strongly stratified fluid consists of quasi-horizontal motions in a sea of weak internal gravity waves. The dynamics, which does not depend on the ambient stratification, is found to be dominated by a balance between the horizontal advective motion and the dissipation due to strong vertical shear. These results can be understood in the framework of the strongly dissipative, small vertical Froude number regime described in Godoy-Diana et al. ${ }^{21}$ and Brethouwer et al. ${ }^{5}$ Some previous studies have looked at stratified turbulence forced by oscillating a grid in a stratified fluid but the main focus was on the dynamics and structure of the turbulent front. ${ }^{22-24}$ Other previous experiments consist in decaying turbulence generated by a grid. ${ }^{6,25-28}$ During the decay, both $R e$ and $F_{h}$ decrease and the turbulence collapses into a strongly stratified flow. However, the time period during which both conditions $F_{h}<1$ and $\mathcal{R}>1$ are fulfilled is short and the flow is rapidly strongly influenced by the viscous effects due to vertical shear. Thus, it remains a challenge to obtain a strongly stratified fully turbulent flow in a laboratory experiment.

As an attempt in this direction, we present here a new experimental set-up of stratified disordered flows forced with columnar vortices. This set-up which is described in Sec. II has allowed us to obtain a constant buoyancy Reynolds number $\mathcal{R}$ during long time periods compared to previous experimental studies of decaying turbulence. The flows are described in details in Sec. III for different buoyancy Reynolds numbers. 


\section{EXPERIMENTAL DESIGN}

\section{A. Experimental setup}

The experiments are carried out in a $1 \mathrm{~m}$ wide, $2 \mathrm{~m}$ long, and $0.6 \mathrm{~m}$ deep tank filled with salt-stratified water. Since the purpose is to investigate strongly stratified turbulence, with a small horizontal Froude number together with the largest possible Reynolds number, we have set the strongest stratification possible with salt: at the surface, the water is fresh with a density equal to 1.0 $\mathrm{g} / \mathrm{cm}^{3}$ and at the bottom of the tank, it is saturated with a density nearly equal to $1.2 \mathrm{~g} / \mathrm{cm}^{3}$ leading to a constant Brunt-Väisälä frequency $N=\sqrt{-\left(g / \rho_{0}\right)(d \bar{\rho} / d z)}$ of $1.7 \mathrm{rad} / \mathrm{s}$ in all experiments, where $g$ is the gravity, $\rho_{0}$ is the mean density, and $\bar{\rho}$ is the basic density profile. The stable linear stratification is established by the well-known two tanks method by means of two computer-controlled volumetric pumps. The density profile is obtained by measuring with a density meter the density of several small samples of fluid taken along the vertical. The comparison between results obtained before and after a set of experiments allowed us to check that the density profile was not modified. The kinematic viscosity $v$ slightly decreases from the bottom to the top from $1.6 \times 10^{-6} \mathrm{~m}^{2} / \mathrm{s}$ to $1.0 \times 10^{-6}$ $\mathrm{m}^{2} / \mathrm{s}$. Throughout the paper, we neglect this variation and consider the viscosity at the middle depth $v \simeq 1.1 \times 10^{-6} \mathrm{~m}^{2} / \mathrm{s}$. The Schmidt number $S c=v / D$ is around 700 , where $D$ is the diffusivity of salt.

As depicted in Figure 1(a), the flow is generated by an arena of 12 dipole generators in the tank. A generator is composed of two symmetric vertical plates whose closing creates a counter-rotating vertical columnar vortex pair (Figure 1(b)) as described by Leweke and Williamson ${ }^{29}$ and Billant and Chomaz. ${ }^{7}$ One motor drives two dipole generators by a system of gears and joints as seen in Figure 1(c). However, the 6 motors are independent and, hence, run with random phases. Thus, each pair of generators produces periodically and independently two dipoles which propagate toward the central part of the tank.

As seen in Figure 1(c), the motor drives a cam with a pulley that pushes two levers fixed on the gears. The particular setting of the levers is chosen such that the dipole generators are closed slowly and smoothly and remain closed during a few seconds. Then, they open slower than for the closing and remains open for some time before closing again. By this way, the dipoles are not perturbed by the subsequent opening of the flaps. In particular, the vortices created during the opening are trapped inside the generators and do not perturb the propagating dipoles. The only control parameter that can

(a)

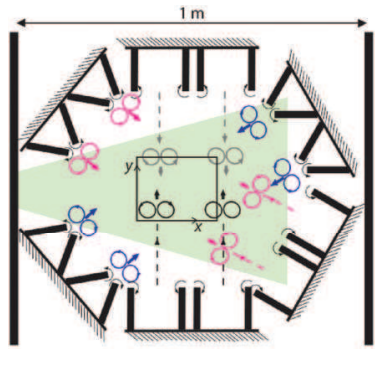

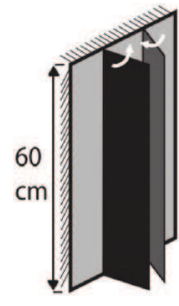

(b)
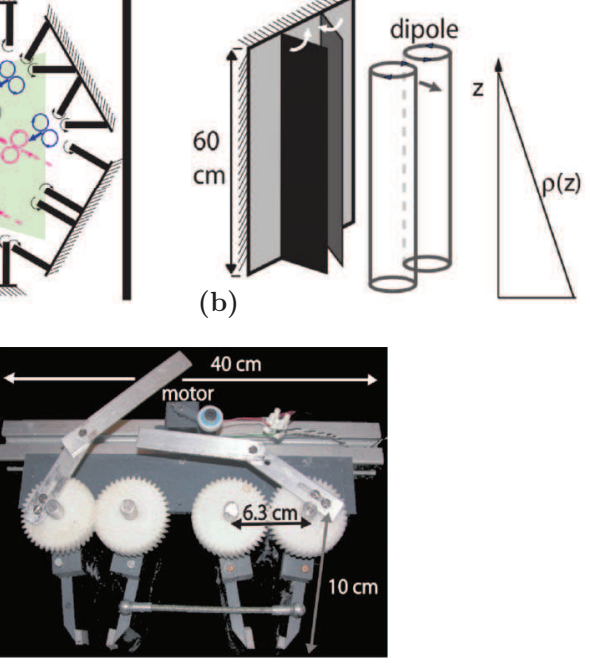

(c)

FIG. 1. Sketch of the experimental apparatus. (a) Top view of the set-up from above. The black rectangle indicates the field of view of the PIV for horizontal cross-sections. (b) Sketch of one dipole generator. (c) View from above of the motor and system driving a pair of vortex generators. 
be varied with this system is therefore the frequency of dipole generation $f_{g}$. When $f_{g}$ is increased, not only the time interval between two successive dipoles decreases but the strength of the dipoles increases as well. The frequency $f_{g}$ has been varied between 0.03 and $0.07 \mathrm{~Hz}$, i.e., one dipole is created by one flap every $14 \mathrm{~s}$ in the fastest cases and every $33 \mathrm{~s}$ in the slowest experiments.

In contrast to other forcing methods (grid turbulence, oscillating tanks) that tend to also force gravity waves, the present forcing forces only vortical motions. Interestingly, this is quite similar to geophysical flows since they are dominated at large and intermediate scales by vortical motions.

Measurements of the velocity field have been obtained by Particle Image Velocimetry (PIV) using FlowMaster software by LaVision. The image acquisition is made by a double-frame camera with resolution of $1280 \times 1024$ pixels. The laser sheet is produced by a $50 \mathrm{~mJ}$ pulsed Nd:YAG dual head laser. As shown in Figure 1(a), the field of view is located in the central part of the fluid volume encircled by the flaps. Velocity fields in vertical cross-section have been also measured by setting the laser sheet vertical. The cross-sections are located approximately at the middle depth. Since the vortex generators are opaque, the laser sheet for the vertical cross-section has to be shined between two vortex generators. In practice, the laser sheet has been placed approximately along the $y$ axis and has been shined between the two generators at the bottom left of Figure 1(a). Therefore, it did not go through the center of the volume encircled by the flaps. The fluid has been seeded with particles made of ion-exchange resin (Purolite). Particles with size $\sim 50 \mu \mathrm{m}$ have been selected by means of filtrations through sieves. By successive density separations, only particles with a density distribution close to the density of the fluid are kept. The particles concentration is a crucial parameter: it should be large enough to achieve good PIV calculations but not too large to prevent a significant attenuation of the laser light. A concentration of approximately 0.1 particles by pixel has been found to be optimal.

The thickness of the light sheet has been adjusted to $3 \mathrm{~mm}$ in the field of view in order to minimize the rate of particles that enter or exit the light sheet between two laser pulses. This improves the accuracy of the PIV calculations. The velocity gradients across the width of the light sheet can lead to erroneous velocity vectors at few points but they are eliminated during the PIV post-processing and thus not used to compute the velocity structure functions. In practice, it is easier to obtain the velocity field in horizontal cross-sections than in vertical ones. First, the mean vertical velocity is smaller than the mean horizontal velocity and second, it is easier to have an homogeneous spreading of particles in horizontal cross-sections than in vertical ones. Thus, the quality of velocity fields is better in the horizontal direction than in the vertical direction. Since the forcing consists only in horizontal motions and the flow is strongly stratified, the vertical velocity is generally sufficiently small so that the variations of the refractive index due to small-scale mixing are weak. Possible effects of the variations of the refractive index are also minimized by calculating the velocity fields used for statistical analysis by means of relatively large interrogation windows of $64 \times 64$ pixels corresponding to physical dimensions of $6.8 \mathrm{~mm}$ for vertical cross-sections and $9.6 \mathrm{~mm}$ for horizontal cross-sections. This implies that the smallest scales of the flow that are of the order of the millimeter are not resolved. The overlapping between the interrogation windows is 50\%, implying that the spatial resolution of the PIV calculation is equal to half the interrogation window size. The statistical quantities have been computed from the raw data without post-processing to fill the missing data (incorrect vectors) by interpolation using the neighbouring correct vectors. For this reason, only structure functions have been computed since there is no data at the locations where erroneous vectors have been eliminated. Spectra have not been computed from the PIV measurements because they require the use of filters to enforce spatial periodicity and also the use of post-processing interpolation to have regularly spaced data. A post-processing treatment has been used to fill the missing vectors only for the figures of the paper showing the velocity fields.

\section{B. Flow characteristics and relevant parameters}

In order to characterize the vortices produced by the vortex generators, we have first carried out several experiments with only one module, i.e., one pair of dipole generator running. Figure 2 shows one example of the flow evolution in a horizontal cross-section starting from a fluid at rest. The first two dipoles move obliquely toward the centre of the tank and toward each other because of 

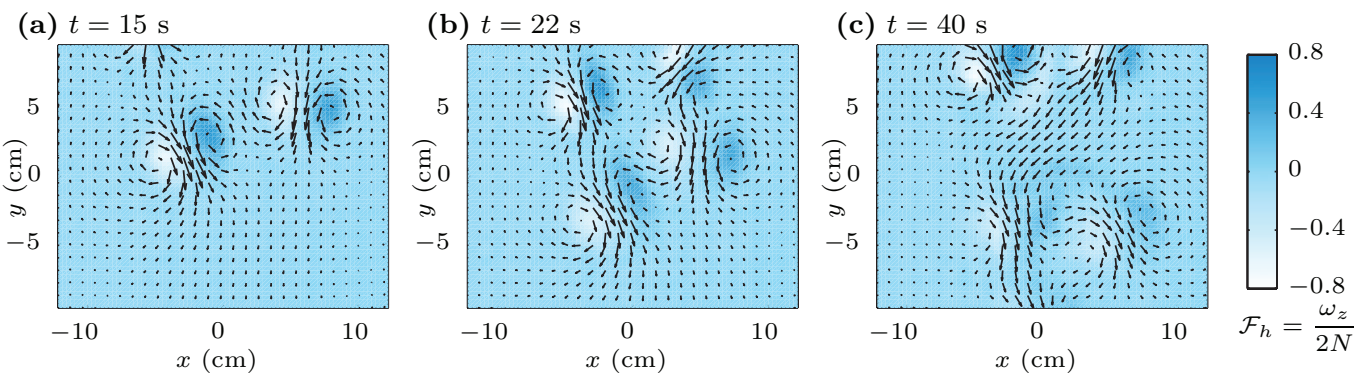

FIG. 2. Horizontal cross-sections of the velocity field (arrows) after switching on one module at $t=0$ from rest. The vector fields are measured at $t=15 \mathrm{~s}, 22 \mathrm{~s}, 40 \mathrm{~s}$. The background shade represents the local horizontal Froude number $\mathcal{F}_{h}=\omega_{z} /(2 N)$, where $\omega_{z}$ is the vertical vorticity. The frequency of injection is $f_{g}=0.05 \mathrm{~Hz}$ and the corresponding non-dimensional numbers associated with the vortices are $R e=290, F_{h}=0.59$, and $\mathcal{R}=100$.

their mutual induction (Figure 2(a)). At $t=22 \mathrm{~s}$ (Figure 2(b)), two new dipoles have been created and start to interact with the first ones. Later on, at $t=40 \mathrm{~s}$, another pair of dipoles has been created, while the first four dipoles have strongly interacted and are disordered.

The characteristics of the dipoles can be obtained by fitting the first vortices that are created (Figure 2(a)) to pair of Lamb-Oseen vortices with vorticity:

$$
\omega_{z}=\sum_{i=1,2} \frac{\Gamma_{i}}{\pi a^{2}} e^{\frac{-\left(x-x_{i}\right)^{2}-\left(y-y_{i}\right)^{2}}{a^{2}}},
$$

where $a$ is the vortex radius, $\Gamma_{i}$ is the circulation, $\left(x_{i}, y_{i}\right)$ is the centre of each vortex. Table I gives the parameters of the dipoles as a function of the frequency of dipole generation $f_{g}$ (i.e., one vortex generator produces one dipole every $1 / f_{g}$ time period). The shape of the dipole, i.e., the vortex radius $a \simeq 18 \mathrm{~mm}$ and the separation distance between the vortices $b=\sqrt{\left(x_{1}-x_{2}\right)^{2}+\left(y_{1}-y_{2}\right)^{2}} \simeq 36$ $\mathrm{mm}$, is almost independent of $f_{g}$ as previously observed by Leweke and Williamson ${ }^{29}$ and Billant and Chomaz. ${ }^{7}$ In contrast, the maximum angular velocity of one vortex $\Omega=\left|\Gamma_{i}\right| /\left(2 \pi a^{2}\right)$ increases approximately linearly with $f_{g}$ as can be seen in Table I. Based upon these parameters, we define the horizontal Froude number and Reynolds number as follows: ${ }^{10}$

$$
F_{h}=\frac{\Omega}{N}, \quad R e=\frac{\Omega a^{2}}{v} .
$$

Since only $\Omega$ varies, the Froude and Reynolds numbers both increase linearly with $f_{g}$ (Table I).

Another convenient local definition of the horizontal Froude number has been also used:

$$
\mathcal{F}_{h}=\frac{\omega_{z}}{2 N},
$$

where $\omega_{z}$ is the vertical vorticity. This local Froude number is shown in Figure 2 as shade contours. As can be seen, its maximum value is around 0.6 in agreement with the corresponding value of $F_{h}$ $=0.59$ for $f_{g}=0.052 \mathrm{~Hz}$ (Table I).

TABLE I. Overview of the parameters characterising the dipoles created by the vortex generators $\left(\Omega, R e, F_{h}\right.$, and $\left.\mathcal{R}\right)$ and the turbulent-like flows in the statistically steady regime $\left(U_{h}, P, R e_{t}, F_{h t}, \mathcal{R}_{t}\right.$, and $\left.\eta=\left(v^{3} / P\right)^{1 / 4}\right)$ as a function of the frequency of generation $f_{g}$.

\begin{tabular}{lcccccccccc}
\hline \hline $\begin{array}{l}f_{g} \\
(\mathrm{~Hz})\end{array}$ & $\begin{array}{c}\Omega \\
(\mathrm{rad} / \mathrm{s})\end{array}$ & $R e$ & $F_{h}$ & $\mathcal{R}$ & $\begin{array}{c}U_{h} \\
(\mathrm{~mm} / \mathrm{s})\end{array}$ & $\begin{array}{c}P \\
\left(\mathrm{~mm}^{2} / \mathrm{s}^{3}\right)\end{array}$ & $R e_{t}$ & $F_{h t}$ & $\mathcal{R}_{t}$ & $\begin{array}{c}\eta \\
(\mathrm{mm})\end{array}$ \\
\hline 0.031 & 0.4 & 120 & 0.24 & 7 & 2.1 & 0.04 & 450 & 0.005 & 0.01 & 2.4 \\
0.035 & 0.55 & 160 & 0.29 & 18 & 2.8 & 0.08 & 700 & 0.006 & 0.02 & 2.0 \\
0.040 & 0.7 & 210 & 0.41 & 35 & 3.4 & 0.15 & 810 & 0.008 & 0.05 & 1.7 \\
0.052 & 1.0 & 290 & 0.59 & 100 & 4.5 & 0.4 & 930 & 0.012 & 0.1 & 1.3 \\
0.062 & 1.27 & 370 & 0.75 & 210 & 5.3 & 0.8 & 900 & 0.017 & 0.25 & 1.1 \\
0.071 & 1.45 & 430 & 0.85 & 310 & 6.0 & 1.2 & 980 & 0.019 & 0.4 & 1.0 \\
\hline \hline
\end{tabular}


The maximum horizontal Froude number $F_{h}$, obtained for the largest frequency $f_{g}$, is equal to 0.85 . This value has to be compared to the threshold $F_{h}=1$ above which the zigzag instability becomes damped by a critical layer. ${ }^{8,9}$ Hence, the value 0.85 ensures that the zigzag instability should be active.

As discussed in the Introduction, another important non-dimensional parameter is the buoyancy Reynolds number $\mathcal{R}=R e F_{h}{ }^{2}{ }^{3,5,14}$ This number measures the importance of viscous effects due to the vertical shear and it controls also the onset of small-scale instabilities. ${ }^{10-12,14}$ As indicated in Table I, the maximum buoyancy Reynolds number is equal to 310 . This value is close but below the threshold $\mathcal{R}_{c}=340$ for the transition to small scales via the development of the shear instability found by Deloncle et al. ${ }^{10}$ for an isolated Lamb-Oseen dipole with $b / a=2.5$. Hence, an isolated dipole should not be subjected to the shear instability. However, the dipoles are not isolated in the present experiments and the interactions among them and the ambient flow will be actually the main source of vertical shear and small-scale instabilities.

In Sec. III, we shall present the results obtained with all the six modules switched on. After a transient flow, the interactions between the periodically produced vortex pairs give rise to a statistically stationary disordered stratified flow. The only difference between the five flows presented is the value of the injection frequency $f_{g}$.

In order to characterize these turbulent-like flows, it is convenient to define also turbulent Froude, Reynolds, and buoyancy Reynolds numbers as

$$
F_{h t}=\frac{P}{U_{h}^{2} N}, R e_{t}=\frac{U_{h}{ }^{4}}{P v}, \text { and } \mathcal{R}_{t}=\frac{P}{v N^{2}},
$$

respectively, where $P$ is the mean energy injection rate, and $U_{h}=\left\langle\left(u_{x}^{2}+u_{y}^{2}\right) / 2\right\rangle_{x y t}^{1 / 2}$ is the square root of the horizontal kinetic energy per unit mass, the brackets $\langle\cdot\rangle_{x y t}$ denote an average over the horizontal cross-section $x, y$, and time $t$. While it is easy to measure the characteristic horizontal velocity $U_{h}$ for each frequency $f_{g}$, the total mean injection rate $P$ needs to be estimated. We consider that 12 columnar dipoles are produced every $1 / f_{g}$ seconds and that the kinetic energy of each dipole spreads over the total volume $H \pi R_{h}{ }^{2}$, where $R_{h} \simeq 0.4 \mathrm{~m}$ is the radius of the fluid volume encircled by the flaps and $H$ is the total water depth. Since the vortices of each dipole are close $(b / a \simeq 2)$, their kinetic energy can be estimated by using the exact expression for the kinetic energy of a Lamb-Chaplygin dipole, ${ }^{30} E_{k}=\pi R^{2} U^{2} H$, where $R$ is the radius of the dipole and $U$ its velocity of propagation in the laboratory frame. Assuming $R \simeq b$ and $U \simeq \Gamma /(2 \pi b)$ with $\Gamma=2 \pi \Omega a^{2}$, we obtain $E_{k}=H \pi a^{2}(a \Omega)^{2}$. These considerations lead to the following approximation for the mean injection rate $P \simeq 12 f_{g}\left(a / R_{h}\right)^{2}(a \Omega)^{2}$. Since the flows are statistically stationary, the total mean injection rate gives an estimate of the order of the total dissipation rate $\varepsilon$. These turbulent non-dimensional numbers are convenient since they can be compared to those employed in numerical studies, such as in Brethouwer et al. ${ }^{5}$ and Augier ${ }^{20}$ Table I summarizes the values of the parameters (4) in our experiments. Most notably, the turbulent buoyancy Reynolds number $\mathcal{R}_{t}$ is much smaller than $\mathcal{R}$ and varies from 0.01 to 0.4 . This is mainly because the turbulent Froude number $F_{h t}$ is typically two orders of magnitude smaller than the Froude number $F_{h}$.

\section{DESCRIPTION OF THE STATISTICALLY STATIONARY STRATIFIED FLOWS}

In this section, we present the results of the experiments with the 12 vortex generators running continuously. We describe qualitatively and quantitatively the flows when the buoyancy Reynolds number is increased.

\section{A. Qualitative description of the flows for increasing buoyancy Reynolds number}

Figure 3 displays horizontal ((a), (c), (e)) and vertical ((b), (d), (f)) cross-sections of the instantaneous velocity fields for three different values of the buoyancy Reynolds number: $\mathcal{R}=7$ in Figs. 3(a) (Multimedia view) and 3(b) (Multimedia view), $\mathcal{R}=100$ in Figs. 3(c) and 3(d), and $\mathcal{R}=310$ in 3(e) (Multimedia view) and 3(f) (Multimedia view). In the horizontal cross-sections 

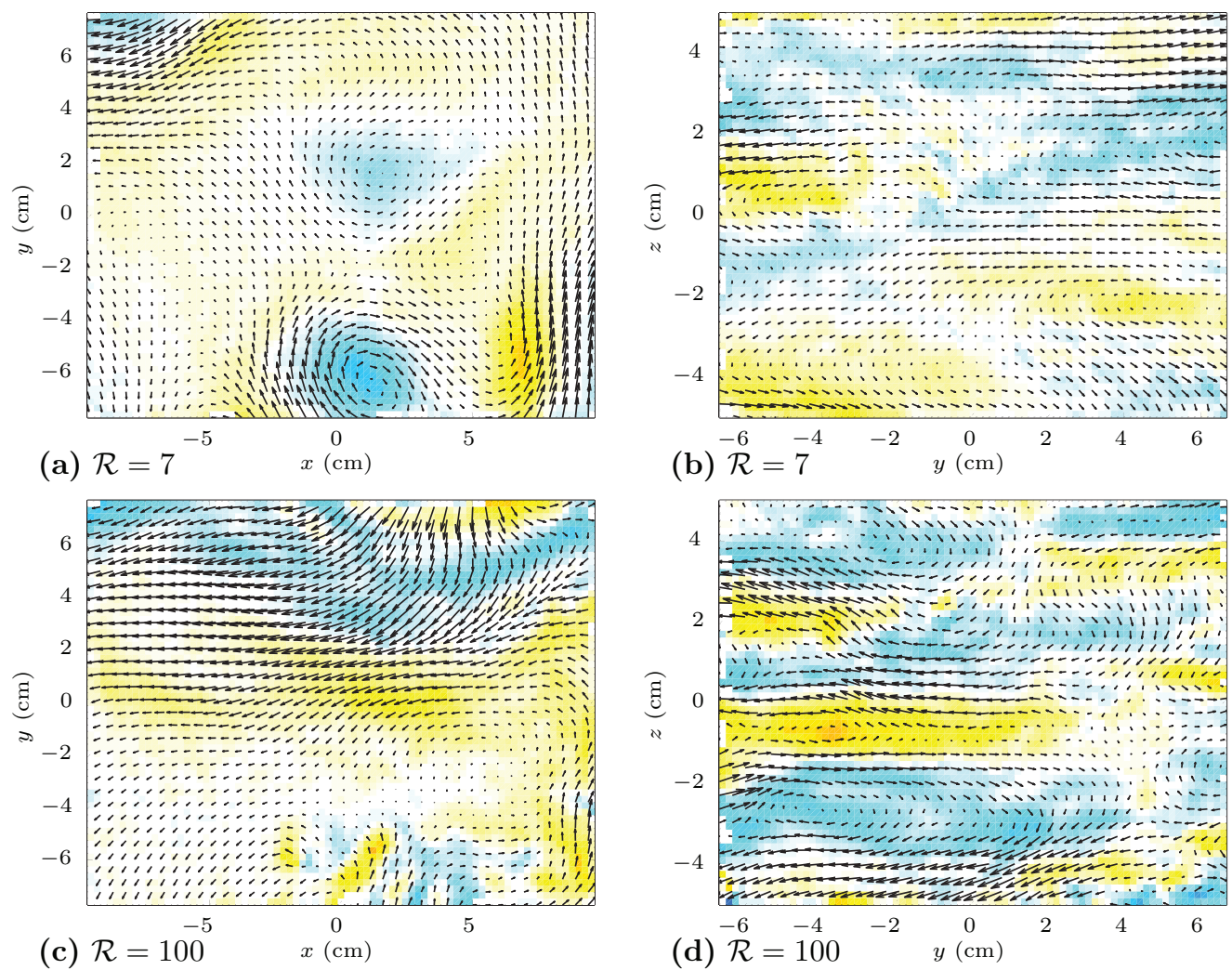

(c) $\mathcal{R}=100$
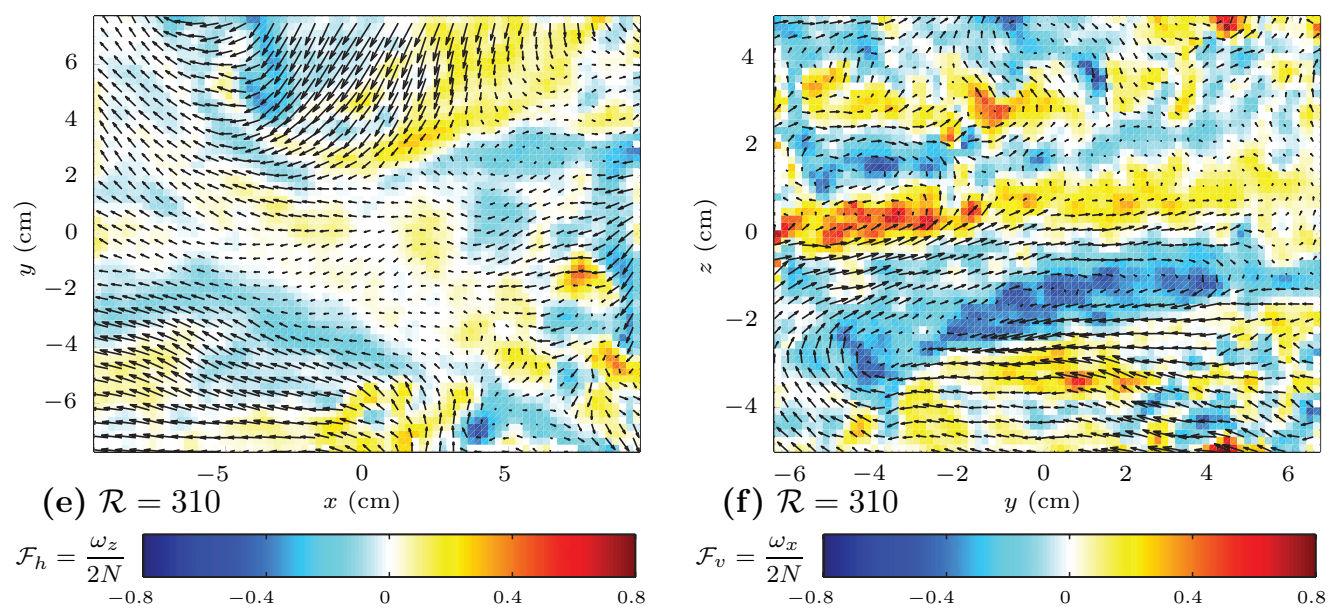

FIG. 3. Horizontal ((a), (c), (e)) and vertical ((b), (d), (f)) cross-sections of the velocity in the quasi-stationary regime for three values of the buoyancy Reynolds number $\mathcal{R}=7$ ((a) and (b)), $\mathcal{R}=100$ ((c) and (d)), and $\mathcal{R}=310$ ((e) and (f)). In the horizontal cross-sections ((a), (c), (e)) the colors show the local horizontal Froude number $\mathcal{F}_{h}$, whereas in the vertical crosssections ((b), (d), (f)) they show the local vertical Froude number $\mathcal{F}_{v}$. Each vector is calculated over a window of $32 \times 32$ pixels (corresponding to PIV resolutions of $4.8 \mathrm{~mm}$ and $3.4 \mathrm{~mm}$ for the horizontal and vertical cross-sections, respectively) with a $50 \%$ overlapping between the windows. Only half of the vectors are shown. See also the movies corresponding to (a), (b), (e), and (f). (Multimedia view) [URL: http://dx.doi.org/10.1063/1.4870703.1] [URL: http://dx.doi.org/10.1063/1.4870703.2] [URL: http://dx.doi.org/10.1063/1.4870703.3] [URL: http://dx.doi.org/10.1063/1.4870703.4].

((a), (c), (e)), the colors show the local horizontal Froude number $\mathcal{F}_{h}=\omega_{z} /(2 N)$, whereas in the vertical cross-sections ((b), (d), (f)) they show the local vertical Froude number $\mathcal{F}_{v}=\omega_{x} /(2 N)$. The field of view is in the central region between the flaps (see Figure 1(a)). 
The horizontal cross-sections show that the flow is dominated by scales typically larger than the injection scale of the forced dipoles and of the order of $U_{h}{ }^{3} / P \sim 20 \mathrm{~cm}$ for all buoyancy Reynolds numbers. However, significant variations are observed when the buoyancy Reynolds number increases. For the smaller buoyancy Reynolds number $\mathcal{R}=7$ (Figure 3(a)), the flow is very smooth. A large scale intense vortex is visible close to $x=0 \mathrm{~cm}$ and $y=-6 \mathrm{~cm}$ (bottom central part) with a negative vertical vorticity patch. In contrast, we see meanders and some smaller scales for $\mathcal{R}=100$ (Figure 3(c)) but no coherent vortices. For the largest buoyancy Reynolds number $\mathcal{R}=310$, large-scale structures with $\left|\mathcal{F}_{h}\right|<0.3$ are still visible but they coexist with smaller structures associated with local horizontal Froude number approaching unity (see in the bottom right corner of Figure 3(e)). These small-scale vortices are produced mainly in the vicinity of the dipole generators. They are due to the fact that, when $\mathcal{R}$ is large, the vortices are destabilized and break into small scales in the vicinity of the vortex generators before reaching the centre of the field of view. In contrast, at small buoyancy Reynolds number, the vortices are not so quickly destabilized and they are able to reach the central part of the tank while still being coherent.

The vertical cross-sections in Figures 3(b), 3(d), and 3(f) show that the flow is strongly threedimensional and organised into horizontal layers for the three buoyancy Reynolds numbers. Interestingly, more and more small-scale structures are superimposed on the layers when the buoyancy Reynolds number is increased. For $\mathcal{R}=310$, these small-scale structures have quite large vertical velocity suggesting the presence of Kelvin-Helmholtz billows.

When the buoyancy Reynolds is increased, the characteristic vertical thickness of the layers also tends to decrease. It is also noteworthy that the extrema of the local vertical Froude number $\mathcal{F}_{v}=\omega_{x} /(2 N)$ increase with $\mathcal{R}$ indicating an increase of the vertical shear. In particular, for the larger buoyancy Reynolds number $\mathcal{R}=310$ (Figure 3(f)), strong shears evidenced by a local vertical Froude number $\mathcal{F}_{v}$ of order unity, are visible in the middle of the cross-sections. Since the Richardson number and the vertical Froude number $\mathcal{F}_{v}$ are related by

$$
R i=\frac{-\left(g / \rho_{0}\right)\left(\partial \rho_{t o t} / \partial z\right)}{\left|\partial \mathbf{u}_{h} / \partial z\right|^{2}} \lesssim \frac{1}{4 \mathcal{F}_{v}^{2}},
$$

with $\rho_{\text {tot }}$ the total density, and where it has been assumed that $-\left(g / \rho_{0}\right)\left(\partial \rho_{t o t} / \partial z\right) \lesssim N^{2}$. We see that a local vertical Froude number $\mathcal{F}_{v}$ of order unity corresponds to a Richardson number of order 1/4, the critical value below which the shear instability could develop for an inviscid parallel stratified flow. This is consistent with the small scale structures seen in Figure 3(f).

\section{B. Evolution of statistical quantities with the buoyancy Reynolds number}

Figure 4 presents normalized probability density functions of the absolute value of the local vertical Froude number $\left|\mathcal{F}_{v}\right|=\left|\omega_{x}\right| /(2 N)$ for different buoyancy Reynolds numbers. As shown by (5), this non-dimensional number is related to the local Richardson number Ri. We see a regular increase of the maximum vertical Froude number when $\mathcal{R}$ is increased. For the highest buoyancy Reynolds number achieved $\mathcal{R}=310$, the maximum value of $\left|\mathcal{F}_{v}\right|$ is of order unity confirming that shear instabilities should occur.

In order to quantify the vertical structure of the flow, we have computed second order transverse vertical structure functions $S_{2 T}(z)=\left\langle\left[\delta u_{y}(z)\right]^{2}\right\rangle$ where $\delta u_{y}(z)=u_{y}\left(\mathbf{x}+z \boldsymbol{e}_{z}\right)-u_{y}(\mathbf{x})$, is the transverse velocity increment. Figure 5 (a) displays the quantity $z^{-2} S_{2 T}(z) /\left(2 U_{y}{ }^{2}\right)$, where $U_{y}=\left\langle u_{y}{ }^{2}\right\rangle_{x y t}^{1 / 2}$, for the five different values of the buoyancy Reynolds number $\mathcal{R}$. At the smallest resolved scales, the velocity signal is smooth so that $\delta u_{y}(z)=u_{y}(\mathbf{x}+z)-u_{y}(\mathbf{x}) \simeq \partial_{z} u_{y}(\mathbf{x}) z$. Thus, the quantity $z^{-2} S_{2 T}(z) /\left(2 U_{y}^{2}\right)$ tends toward a constant equal to $1 / L_{v}{ }^{2}$, where $L_{v}$ is equivalent to the so-called microscale $L_{v}=\left(2 U_{y}{ }^{2} /\left\langle\left[\partial_{z} u_{y}\right]^{2}\right\rangle\right)^{1 / 2}$. We see that the quantity $1 / L_{v}{ }^{2}$ shown by the crosses in Figure 5(a) varies by a factor two with $\mathcal{R}$. Even if the smallest dissipative scales are not resolved, the quantity $L_{v}$ is almost equivalent to the real microscale of the flow since $z^{-2} S_{2 T}(z) /\left(2 U_{y}{ }^{2}\right)$ tends to a constant for small $z$ (Figure 5(a)). Hence, this length scale is expected to reliably characterize the vertical structure of the flow. The evolution of this characteristic length scale when $\mathcal{R}$ is varied is shown in Figures 5(b) and 5(c). Following Brethouwer et al., ${ }^{5}$ two different scaling are tested: a viscous 


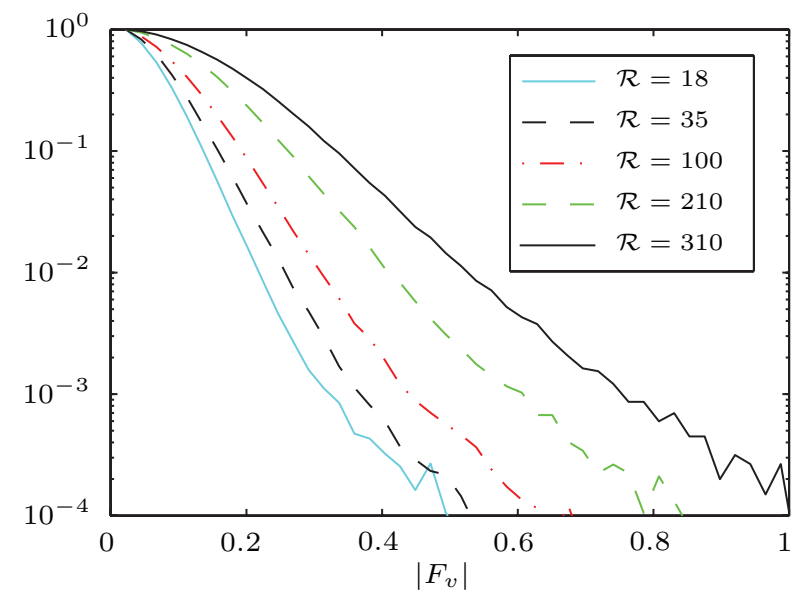

FIG. 4. Normalized probability density function of the local vertical Froude number $\left|\mathcal{F}_{v}\right|=\left|\omega_{x}\right| /(2 N) \simeq(4 R i)^{-1 / 2}$ for various $\mathcal{R}$ as indicated in the inset. Each curve is normalized by its maximum value.

scaling in Figure 5(b) and an inviscid scaling in Figure 5(c). The viscous scaling is obtained by balancing the dissipation due to vertical gradients and the horizontal advection $L_{v} \sim \sqrt{v a / U} \sim a / \sqrt{R e} .{ }^{21}$ The inviscid scaling is obtained by balancing the buoyancy term to the horizontal advection so that $L_{v} \sim U / N \sim a F_{h}{ }^{3}$ Even if there are only five values of $\mathcal{R}$, the vertical scale (Figure 5(b)) seems to follow the viscous scaling $\left(L_{v} / a\right) R e^{1 / 2}$ at least up to $\mathcal{R}=200$. Inversely, the vertical scale (Figure 5(c)) seems to asymptote to the inviscid scaling $L_{v} /\left(a F_{h}\right)$ when $\mathcal{R}$ increases. It is consistent with the interpretation of a progressive evolution as the buoyancy Reynolds number increases from a viscous regime in which the vertical length scale is fixed by viscous effects, ${ }^{21}$ to a nonlinear stratified regime for which the vertical length scale is the buoyancy length scale. ${ }^{3}$
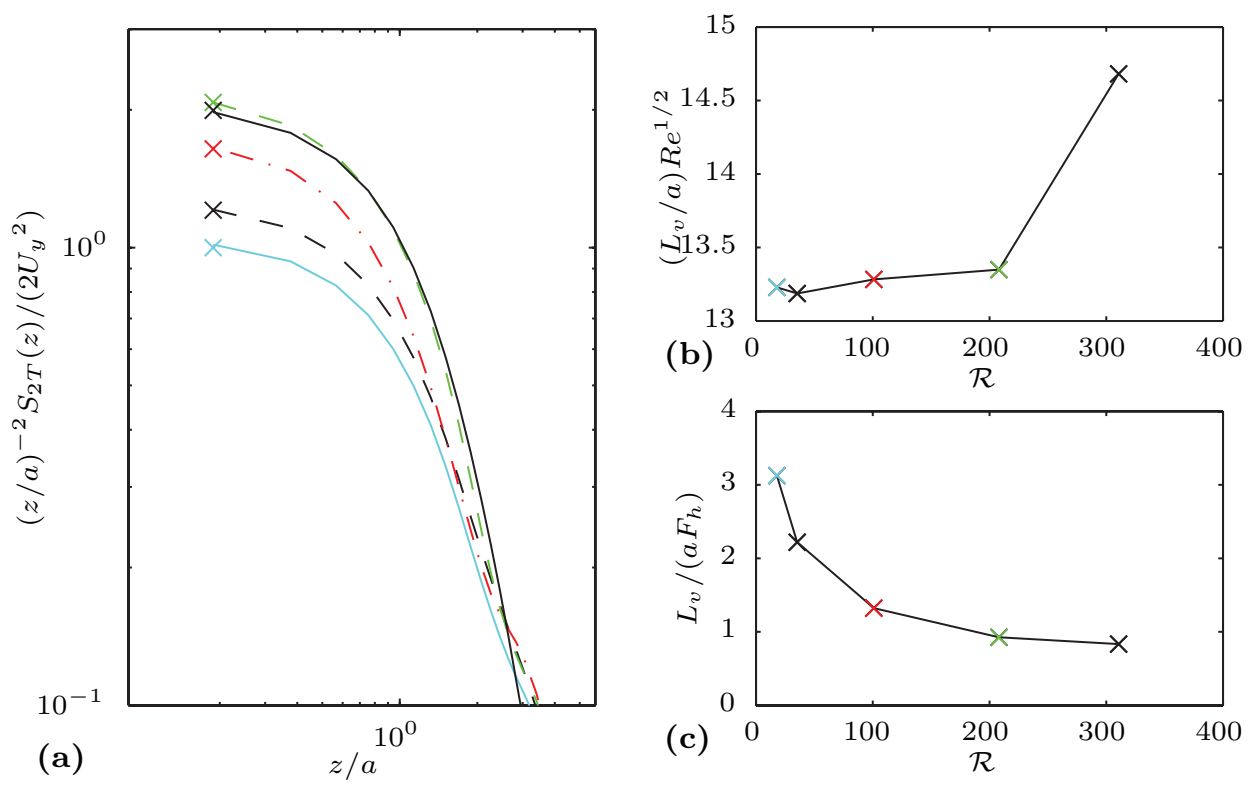

FIG. 5. Variation of the vertical length scale $L_{v}$ with $\mathcal{R}$. (a) Viscously compensated vertical structure function of order two $(z / a)^{-2} S_{2 T}(z) /\left(2 U_{y}{ }^{2}\right)$ for different $\mathcal{R}$ (see Figure 4 for the values and representations). The crosses correspond to the values used to compute $L_{v}$ for each $\mathcal{R}$. (b) rescaled characteristic length scale $\left(L_{v} / a\right) R e^{1 / 2}$ and (c) $L_{v} /\left(a F_{h}\right)$ as a function of $\mathcal{R}$. 

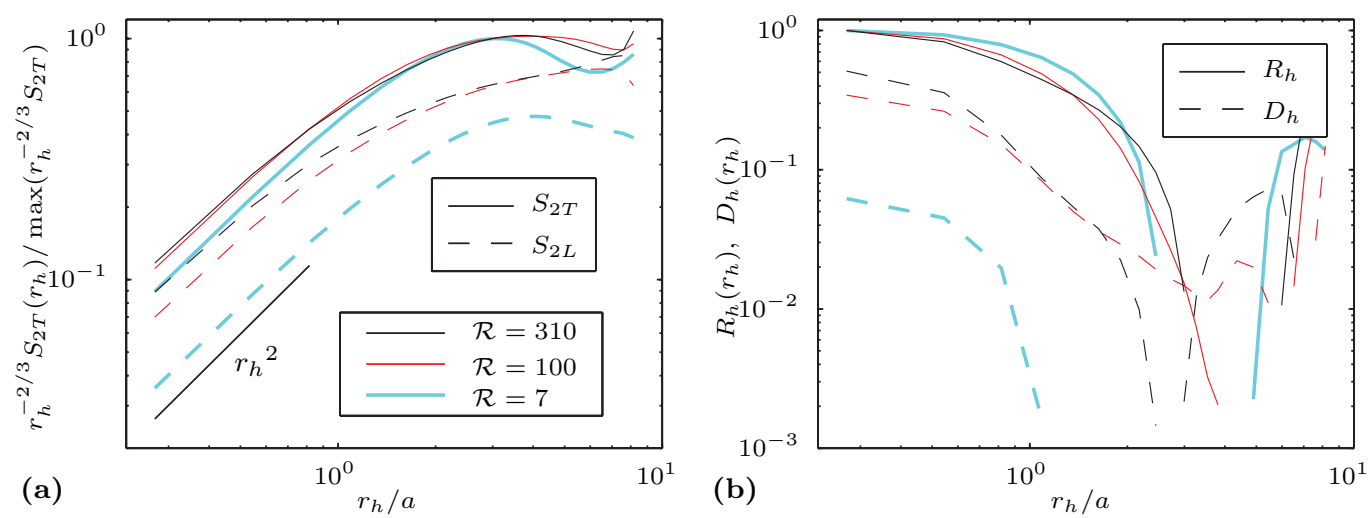

FIG. 6. (a) Compensated transverse and longitudinal horizontal second order structure functions for different $\mathcal{R}$. (b) Estimations of the correlations of vertical vorticity $R_{h}\left(r_{h}\right)$ and of horizontal divergence $D_{h}\left(r_{h}\right)$ normalized by $\max \left(R_{h}\left(r_{h}\right)\right)$.

The horizontal velocity fields have been used to compute the horizontal transverse second order structure function $S_{2 T}\left(r_{h}\right)=\left\langle\left[\delta u_{x}\left(\delta y=r_{h}\right)\right]^{2}+\left[\delta u_{y}\left(\delta x=r_{h}\right)\right]^{2}\right\rangle / 2$ and the horizontal longitudinal second order structure function $S_{2 L}\left(r_{h}\right)=\left\langle\left[\delta u_{x}\left(\delta x=r_{h}\right)\right]^{2}+\left[\delta u_{y}\left(\delta y=r_{h}\right)\right]^{2}\right\rangle / 2$, where $\delta u_{x}(\delta y)=$ $u_{x}\left(\mathbf{x}+\delta y \boldsymbol{e}_{\boldsymbol{y}}\right)-u_{x}(\mathbf{x})$ is the transverse increment of $u_{x}, \delta u_{x}(\delta x)=u_{x}\left(\mathbf{x}+\delta x \boldsymbol{e}_{x}\right)-u_{x}(\mathbf{x})$ is the longitudinal increment of $u_{x}$, and the increments of $u_{y}$ are defined similarly. The compensated longitudinal and transverse structure functions $r_{h}^{-2 / 3} S_{2 T}$ and $r_{h}^{-2 / 3} S_{2 L}$ are plotted in Figure 6(a) for three buoyancy Reynolds numbers: $\mathcal{R}=7$ in black, $\mathcal{R}=100$ in dark and $\mathcal{R}=310$ in light. A large inertial range would be associated with structure functions scaling like $r_{h}{ }^{2 / 3}$, thus leading to a flat slope within a range of scales in Figure 6(a). The power law at the smaller scales correspond to a $r_{h}^{-2}$ slope (shown by the black straight line) since $S_{2 T}\left(r_{h}\right) \simeq\left\langle\left[\partial_{y} u_{x}\right]^{2}+\left[\partial_{x} u_{y}\right]^{2}\right\rangle r_{h}{ }^{2} / 2$ for small $r_{h}$ (and similarly for $S_{2 L}$ ). When $\mathcal{R}$ increases, we see a flattening of the compensated structure functions at very large scales and the compensated structure functions become less steep at the scales $r_{h} \sim a$ and there is an increase of the energy at the small scales, particularly in the longitudinal structure functions. Although the compensated structure functions do not exhibit a clear flat plateau, the decrease of their slope when $\mathcal{R}$ increases could be the first indication of the inertial range that is predicted for large $\mathcal{R} .{ }^{5}$ Remarkably, the variations with the buoyancy Reynolds number are much more important for the longitudinal structure function (dashed curves) than for the transverse structure function (continuous curves). At large scales, $S_{2 T}$ dominates $S_{2 L}$ because the flow is forced by columnar vertical vortices. However, $S_{2 L}$ strongly increases at small scales when $\mathcal{R}$ is varied.

In order to interpret this effect, it is convenient to consider correlations of horizontal divergence $D_{h}\left(r_{h}\right)=\left\langle\nabla_{h} \cdot \mathbf{u}_{h}(\mathbf{x}) \nabla_{h} \cdot \mathbf{u}_{h}\left(\mathbf{x}+\mathbf{r}_{h}\right)\right\rangle_{\mathbf{x}}$ and of vertical vorticity $R_{h}\left(r_{h}\right)=\left\langle\omega_{z}(\mathbf{x}) \omega_{z}\left(\mathbf{x}+\mathbf{r}_{h}\right)\right\rangle_{\mathbf{x}}$, where $r_{h}=\left|\mathbf{r}_{h}\right|$. Lindborg ${ }^{31}$ has shown that for an axisymmetric, incompressible, homogeneous flow, these correlations are related to the structure functions of horizontal velocity through the symmetric relations

$$
\begin{aligned}
D_{h} & =-\frac{1}{2}\left[\frac{1}{r_{h}} \frac{\partial S_{2 T}}{\partial r_{h}}-\frac{1}{r_{h}{ }^{2}} \frac{\partial}{\partial r_{h}}\left(r_{h}{ }^{2} \frac{\partial S_{2 L}}{\partial r_{h}}\right)\right], \\
R_{h} & =-\frac{1}{2}\left[\frac{1}{r_{h}} \frac{\partial S_{2 L}}{\partial r_{h}}-\frac{1}{r_{h}{ }^{2}} \frac{\partial}{\partial r_{h}}\left(r_{h}{ }^{2} \frac{\partial S_{2 T}}{\partial r_{h}}\right)\right] .
\end{aligned}
$$

The correlations $D_{h}$ and $R_{h}$ computed from the structure functions by means of (6) and (7) are plotted in Figure 6(b). Since the assumptions of axisymmetry and homogeneity are only (approximately) valid at relatively small scales, the results at the large scales are meaningless. At separation scales $r_{h} \lesssim 2 a$, the correlation of vertical vorticity $R_{h}$ dominates the correlation of horizontal divergence $D_{h}$. For $\mathcal{R}=7, D_{h}$ is very small showing that the horizontal flow is non-divergent as in twodimensional flows. When $\mathcal{R}$ increases, $D_{h}$ strongly increases at the smaller scales up to half the vorticity correlation $R_{h}$ indicating that the dynamics is more three-dimensional. 


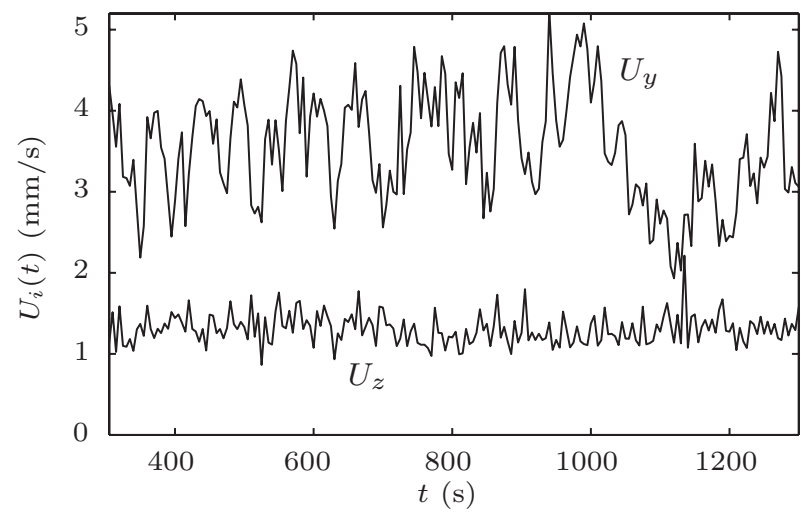

FIG. 7. Horizontal and vertical averaged velocities as a function of time for $\mathcal{R}=310$. For each time the velocities are obtained by averaging over the total vertical cross-section.

\section{Steadiness and inhomogeneity of the flow}

In order to check whether the flow is statistically stationary, the mean kinetic energy has been measured over long period of time. For example, Figure 7 shows the rms of the horizontal and vertical velocities over the vertical cross-section $y, z: U_{y}=\left\langle u_{y}{ }^{2}\right\rangle_{y z}^{1 / 2}$ and $U_{z}=\left\langle u_{z}{ }^{2}\right\rangle_{y z}^{1 / 2}$ as a function of time. The recording starts $5 \mathrm{~min}$ after switching on the vortex generators and last more than 15 $\min$. No mean trend is visible in the time evolution of the averaged horizontal and vertical velocities. Both mean velocity components seem to be statistically stationary with high fluctuations typical of turbulent flows. The mean vertical velocity is three times smaller than the mean horizontal velocity despite that the horizontal Froude number is of order unity $\left(F_{h}=0.85\right)$.

Similarly, in order to check the spatial homogeneity of the flow, Figure 8(a) shows the mean (or more precisely "low pass filtered") flow $\mathbf{U}_{h}=\left\langle\mathbf{u}_{h}\right\rangle_{t}$ in a horizontal cross-section obtained by averaging over 100 instantaneous velocity fields with a frequency of acquisition of $0.5 \mathrm{~Hz}$ (i.e., over a time period of $200 \mathrm{~s}$ ). The vectors correspond to the mean flow and the blue contours represent the rms of velocity fluctuations $\left\langle\left|\mathbf{u}_{h}-\mathbf{U}_{h}\right|^{2} / 2\right\rangle_{t}^{1 / 2}$. The mean flow is non-zero with maximum values around $12 \mathrm{~mm} / \mathrm{s}$. Such mean velocity is quite large since the instantaneous velocity is of the order of $20 \mathrm{~mm} / \mathrm{s}$. The mean flow goes in the negative $x$ direction toward the zone left open between the vortex generators in order to shine the laser sheet (see Figure 1(a)). This mean flow is also visible in the instantaneous velocity fields (Figures 3(a), 3(c), and 3(e)). The velocity fluctuations are also inhomogeneous with rms values approximately twice larger near the dipole generators than in the
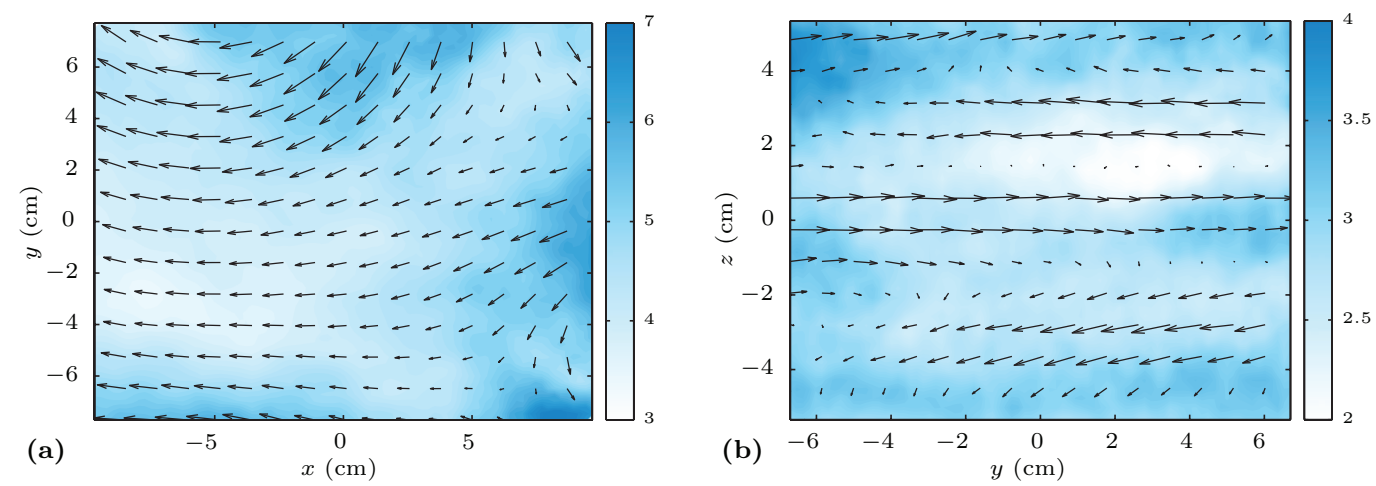

FIG. 8. Horizontal (a) and vertical (b) cross-sections of mean flow $\mathbf{U}_{/ /}=\left\langle\mathbf{u}_{/ /}\right\rangle_{t}$ (arrows) and rms of the velocity fluctuations $\left\langle\left|\mathbf{u}_{/ /}-\mathbf{U}_{/ /}\right|^{2} / 2\right\rangle_{t}^{1 / 2}$ (background shade, in $\mathrm{mm} / \mathrm{s}$ ) for $\mathcal{R}=310$, where $\mathbf{u}_{/ /}$is the velocity parallel to the cross-section. The maximum mean velocity is $12 \mathrm{~mm} / \mathrm{s}$ in (a) and $4 \mathrm{~mm} / \mathrm{s}$ in (b). 
central region. This inhomogeneity is a drawback of the present forced flow compared to decaying grid turbulence. The maximum rms of velocity fluctuations is approximately twice smaller than the maximum mean flow velocity. Figure 8(b) presents the same type of velocity decomposition but in a vertical cross-section. Horizontal flow in alternating direction and vertically sheared are clearly visible. This indicates that the layers evolve slowly and stay during long periods at the same vertical position. The mean velocity in the $y$-direction has maximum values of $4 \mathrm{~mm} / \mathrm{s}$ which is smaller than the mean velocity in the $x$-direction as already seen in the horizontal cross-section (Figure 8(a)). Measurements over long period of time show that these layers evolve over a very long time scale, typically several minutes. Such mean flow with large horizontal scales, vertical scales close to the buoyancy length scale, and slow evolution resembles the shear modes observed in numerical simulations. ${ }^{19}$

\section{SUMMARY AND CONCLUSIONS}

We have set-up a novel experiment of continuously forced, strongly stratified disordered flow so as to approach the strongly stratified turbulent regime existing for large buoyancy Reynolds number. This experiment differs from previous experiments on decaying stratified turbulence since the forcing consists in vertically invariant columnar vortex pairs generated intermittently.

After an initial spontaneous three-dimensionalisation of the flow, a statistically stationary disordered state is reached. The Brunt-Väisälä frequency has been set to its maximum value while the frequency of dipole generation and circulation of the vortices have been varied so as to explore the ranges of horizontal Froude number and buoyancy Reynolds number: $0.24<F_{h}<0.85$ and $7<\mathcal{R}<310$.

The flow exhibits thin horizontal layers associated with relatively strong vertical gradients. When the buoyancy Reynolds number $\mathcal{R}$ is increased, we observe a progressive evolution. For low $\mathcal{R}$ (and $F_{h}$ ), the flow is quasi-horizontal and the structures are smooth. This is consistent with the viscosity dominated stratified regime which occurs for small $\mathcal{R} .^{5,21}$ In contrast, for the largest $\mathcal{R}$ achieved, we observe small scale structures superimposed on the large scale horizontal layers, suggesting that these experiments approach the strongly stratified turbulent regime. ${ }^{5}$ Consistently, the local vertical Froude number $\mathcal{F}_{v} \equiv \omega_{x} /(2 N)$ is of order unity. Vertical cross-sections show that small-scale billows are common features of the flow. However, the horizontal second order structure functions compensated by $r_{h}^{2 / 3}$ do not exhibit any clear flat range when $\mathcal{R}$ is increased but only a flattening consistent with weak nonlinear transfers toward small scales. Actually, this is not too surprising since the maximum turbulent buoyancy Reynolds number is still quite small $\mathcal{R}_{t} \simeq 0.4$ compared to the values $\mathcal{R}_{t}=O(10)$ achieved in the numerical simulations where an inertial range has been observed. ${ }^{5}$ Nevertheless, we point out that the present experiments have Schmidt number values similar to those in the oceans since salt is used as stratifying agent. This is in contrast with DNS of stratified turbulence where the Schmidt number is generally set to unity.

Hence, these experimental results need to be supported and extended. This can be done numerically and experimentally. In a recent study, ${ }^{20}$ we have numerically reproduced the experimental flows with a pseudo-spectral code and a forcing very similar to the experimental one. These numerical results recover the present experimental results and extend them toward higher $\mathcal{R}$ keeping $F_{h}$ low, hence, toward the strongly stratified fully turbulent regime. In particular, they show that the buoyancy Reynolds number needs to be increased to $\mathcal{R}=2000$ to start to see an inertial range in the horizontal spectra of kinetic energy.

Experiments with a larger size could be also carried out. Indeed, the present experimental apparatus has a size $\sim 1 \mathrm{~m}$ and it could be scaled up using large facilities. It seems feasible to gain a factor 5 with vortices of radius $\sim 10 \mathrm{~cm}$. Since the buoyancy Reynolds number $\mathcal{R}=\operatorname{Re} F_{h}{ }^{2}=$ $\Omega^{3} a^{2} /\left(v N^{2}\right)$ goes like $a^{2}$ if $\Omega$ and $N$ are kept constant, it should be possible to overcome the viscous doom of stratified experiments and to produce fully strongly stratified turbulence with $\mathcal{R}=O(8000)$ or, equivalently, with a turbulent buoyancy Reynolds number $\mathcal{R}_{t}$ of order 10, i.e., equivalent to the highest buoyancy Reynolds number simulated by Brethouwer et al. ${ }^{5}$ 


\section{ACKNOWLEDGMENTS}

We are indebted to the SPEA team of Météo-France, in particular to J. C. Boulay who designed and made the flaps, and to A. Garcia of LadHyX who assembled them. We thank F. Moisy for helpful discussions.

${ }^{1}$ J. J. Riley and E. Lindborg, "Stratified turbulence: A possible interpretation of some geophysical turbulence measurements," J. Atmos. Sci. 65, 2416-2424 (2008).

${ }^{2}$ J. J. Riley and M.-P. Lelong, "Fluid motions in the presence of strong stable stratification," An. Rev. Fluid Mech. 32, 613-657 (2000).

${ }^{3}$ P. Billant and J.-M. Chomaz, "Self-similarity of strongly stratified inviscid flows," Phys. Fluids 13, 1645-1651 (2001).

${ }^{4}$ D. A. Hebert and S. M. de Bruyn Kops, "Predicting turbulence in flows with strong stable stratification," Phys. Fluids 18, 066602 (2006).

${ }^{5}$ G. Brethouwer, P. Billant, E. Lindborg, and J.-M. Chomaz, "Scaling analysis and simulation of strongly stratified turbulent flows," J. Fluid Mech. 585, 343-368 (2007).

${ }^{6}$ O. Praud, A. M. Fincham, and J. Sommeria, "Decaying grid turbulence in a strongly stratified fluid," J. Fluid Mech. 522, $1-33$ (2005)

${ }^{7}$ P. Billant and J.-M. Chomaz, "Experimental evidence for a new instability of a vertical columnar vortex pair in a strongly stratified fluid," J. Fluid Mech. 418, 167-188 (2000).

${ }^{8}$ P. Billant, "Zigzag instability of vortex pairs in stratified and rotating fluids. Part 1. General stability equations," J. Fluid Mech. 660, 354-395 (2010).

${ }^{9}$ P. Billant, A. Deloncle, J.-M. Chomaz, and P. Otheguy, "Zigzag instability of vortex pairs in stratified and rotating fluids. Part 2. Analytical and numerical analyses," J. Fluid Mech. 660, 396-429 (2010).

${ }^{10}$ A. Deloncle, P. Billant, and J.-M. Chomaz, "Nonlinear evolution of the zigzag instability in stratified fluids: A shortcut on the route to dissipation," J. Fluid Mech. 599, 229-238 (2008).

${ }^{11}$ M. L. Waite and P. K. Smolarkiewicz, "Instability and breakdown of a vertical vortex pair in a strongly stratified fluid," J. Fluid Mech. 606, 239-273 (2008).

${ }^{12}$ P. Augier and P. Billant, "Onset of secondary instabilities on the zigzag instability in stratified fluids," J. Fluid Mech. 682, 120-131 (2011).

${ }^{13}$ E. Lindborg, "The energy cascade in a strongly stratified fluid," J. Fluid Mech. 550, 207-242 (2006).

${ }^{14}$ J. J. Riley and S. M. de Bruyn Kops, "Dynamics of turbulence strongly influenced by buoyancy," Phys. Fluids 15, 2047-2059 (2003).

${ }^{15}$ M. L. Waite and P. Bartello, "Stratified turbulence dominated by vortical motion," J. Fluid Mech. 517, 281-308 (2004).

${ }^{16}$ E. Lindborg and G. Brethouwer, "Stratified turbulence forced in rotational and divergent modes," J. Fluid Mech. 586, 83-108 (2007).

${ }^{17}$ M. L. Waite, "Stratified turbulence at the buoyancy scale," Phys. Fluids 23, 066602 (2011).

${ }^{18}$ P. Augier, J.-M. Chomaz, and P. Billant, "Spectral analysis of the transition to turbulence from a dipole in stratified fluids," J. Fluid Mech. 713, 86-108 (2012).

${ }^{19}$ L. M. Smith and F. Waleffe, "Generation of slow large scales in forced rotating stratified turbulence," J. Fluid Mech. 451, 145-168 (2002).

${ }^{20}$ P. Augier, "Turbulence in strongly stratified fluids: Cascade processes," Ph.D. thesis (LadHyX, Ecole Polytechnique, 2011), http://tel.archives-ouvertes.fr/tel-00697245.

${ }^{21}$ R. Godoy-Diana, J.-M. Chomaz, and P. Billant, "Vertical length scale selection for pancake vortices in strongly stratified viscous fluids," J. Fluid Mech. 504, 229-238 (2004).

${ }^{22} \mathrm{~S}$. Thorpe, "On the layers produced by rapidly oscillating a vertical grid in a uniformly stratified fluid," J. Fluid Mech. 124, 391-409 (1982).

${ }^{23}$ E. J. Hopfinger, “Turbulence in stratified fluids: A review,” J. Geophys. Res. 92, 5287-5303, doi:10.1029/JC092iC05p05287 (1987).

${ }^{24}$ F. K. Browand, D. Guyomar, and S.-C. Yoon, "The behavior of a turbulent front in a stratified fluid: Experiments with an oscillating grid,” J. Geophys. Res., [Oceans] 92, 5329-5341, doi:10.1029/JC092iC05p05329 (1987).

${ }^{25}$ E. C. Itsweire, K. Helland, and C. W. Vanatta, "The evolution of grid-generated turbulence in a stably stratified fluid," J. Fluid Mech. 162, 299-338 (1986).

${ }^{26}$ J. H. Lienhard and C. W. Van Atta, "The decay of turbulence in thermally stratified flow," J. Fluid Mech. 210, 57-112 (1990).

${ }^{27}$ K. H. Yoon and Z. Warhaft, "The evolution of grid-generated turbulence under conditions of stable thermal stratification," J. Fluid Mech. 215, 601-638 (1990).

${ }^{28}$ A. M. Fincham, T. Maxworthy, and G. R. Spedding, "Energy dissipation and vortex structure in freely decaying, stratified grid turbulence," Dyn. Atmos. Oceans 23, 155-169 (1996).

${ }^{29}$ T. Leweke and C. H. T. Williamson, "Cooperative elliptic instability of a vortex pair," J. Fluid Mech. 360, 85-119 (1998).

${ }^{30}$ V. Meleshko and G. Heijst, "On chaplygin's investigations of two-dimensional vortex structures in an inviscid fluid," J. Fluid Mech. 272, 157-182 (1994).

${ }^{31}$ E. Lindborg, "Horizontal wavenumber spectra of vertical vorticity and horizontal divergence in the upper troposphere and lower stratosphere," J. Atmos. Sci. 64, 1017-1025 (2007). 